Bull. Korean Math. Soc. 51 (2014), No. 1, pp. 189-206

http://dx.doi.org/10.4134/BKMS.2014.51.1.189

\title{
ON SOLVABILITY OF THE DISSIPATIVE KIRCHHOFF EQUATION WITH NONLINEAR BOUNDARY DAMPING
}

\author{
ZAI-YUN ZhANG AND JiAN-HUA HUANG
}

ABSTRACT. In this paper, we prove the global existence and uniqueness of the dissipative Kirchhoff equation

$$
\begin{aligned}
& u_{t t}-M\left(\|\nabla u\|^{2}\right) \triangle u+\alpha u_{t}+f(u)=0 \text { in } \Omega \times[0, \infty), \\
& u(x, t)=0 \text { on } \Gamma_{1} \times[0, \infty), \\
& \frac{\partial u}{\partial \nu}+g\left(u_{t}\right)=0 \text { on } \Gamma_{0} \times[0, \infty), \\
& u(x, 0)=u_{0}, u_{t}(x, 0)=u_{1} \text { in } \Omega
\end{aligned}
$$

with nonlinear boundary damping by Galerkin approximation benefited from the ideas of Zhang et al. [33]. Furthermore,we overcome some difficulties due to the presence of nonlinear terms $M\left(\|\nabla u\|^{2}\right)$ and $g\left(u_{t}\right)$ by introducing a new variables and we can transform the boundary value problem into an equivalent one with zero initial data by argument of compacity and monotonicity.

\section{Introduction}

Let $\Omega$ be a bounded domain of $R^{n}$ and let $\Gamma$ denote its $C^{2}$ boundary. Assume that $\Gamma$ consists of two parts, $\Gamma_{0}$ and $\Gamma_{1}$, with positive measure and such that $\Gamma_{0}$ and $\Gamma_{1}$ are closed and disjoint. Let $\nu=\left(\nu_{1}, \nu_{2}, \ldots, \nu_{n}\right)$ denote the unit outward normal to $\Gamma$ and let $\frac{\partial}{\partial \nu}$ denote the normal derivative.

This paper is concerned with the global existence and uniqueness of solutions of the following dissipative Kirchhoff equation with nonlinear boundary damping

$$
\left\{\begin{array}{l}
u_{t t}-M\left(\|\nabla u\|^{2}\right) \triangle u+\alpha u_{t}+f(u)=0 \text { in } \Omega \times[0, \infty), \\
u(x, t)=0 \text { on } \Gamma_{1} \times[0, \infty), \\
\frac{\partial u}{\partial \nu}+g\left(u_{t}\right)=0 \text { on } \Gamma_{0} \times[0, \infty), \\
u(x, 0)=u^{0}(x), u_{t}(x, 0)=u^{1}(x) \text { in } \Omega,
\end{array}\right.
$$

where $\|u\|^{2}=\int_{\Omega}|u|^{2} d x, \alpha>0$ and $M(s), f(u), g\left(u_{t}\right)$ are functions enjoying some properties (see (A1)-(A3) below).

Received January 31, 2013; Revised April 5, 2013.

2010 Mathematics Subject Classification. 35B40, 35L70.

Key words and phrases. global existence, dissipative Kirchhoff equation, Galerkin approximation, boundary damping. 
For the case, $M(s)=1$, we have the following wave equation with Dirichlet's boundary conditions

$$
\left\{\begin{array}{l}
u_{t t}-\triangle u+\alpha u_{t}+f(u)=0 \text { in } \Omega \times[0, \infty), \\
u(x, t)=0 \text { on } \partial \Omega \times[0, \infty), \\
u(x, 0)=u^{0}(x), u_{t}(x, 0)=u^{1}(x) \text { in } \Omega .
\end{array}\right.
$$

The problem (1.1) arises in many fields such as classical mechanics, fluid dynamics, quantum field theory (see $[27,33]$ ). Later, lots of researchers obtain global existence and uniqueness of solution of the equation (1.2) (see $[8,9,10,15,24,25,26,28])$. It is worth mentioning that Z. Y. Zhang et al. [33] recently have investigated global existence and uniform decay for wave equation with dissipative term and boundary damping as follows

$$
\left\{\begin{array}{l}
u_{t t}-\Delta u+b(x) u_{t}+f(u)=0 \text { in } \Omega \times[0, \infty) \\
u=0 \text { on } \Gamma_{1} \times[0, \infty) \\
\frac{\partial u}{\partial \nu}+g\left(u_{t}\right)=0 \text { on } \Gamma_{0} \times[0, \infty) \\
u(x, 0)=u^{0}(x), u_{t}(x, 0)=u^{1}(x) \text { in } \Omega
\end{array}\right.
$$

under some assumptions on nonlinear feedback function $g\left(u_{t}\right)$. They have obtain the results by means of Galerkin method and the multiplier technique. More precisely, they introduced a new variables and transformed the boundary value problem into an equivalent one with zero initial data by argument of compacity and monotonicity. If $M(s)=1$ and $\alpha=b(x)$, then the above problem reduce to problem (1.2). More details are present in [33]. Later on, Zhang et al. [32] studied the wellposedness and uniform stability of strong and weak solutions of the nonlinear generalized dissipative Klein-Gordon equation with nonlinear damped boundary conditions given by

$$
\left\{\begin{array}{l}
u_{t t}-\Delta u+b(x) u_{t}+f(u)+h(\nabla u)=0 \text { in } \Omega \times(0, \infty), \\
u=0 \text { on } \Gamma_{0} \times(0, \infty) \\
\frac{\partial u}{\partial \nu}+g\left(u_{t}\right)=0 \text { on } \Gamma_{1} \times(0, \infty) \\
u(x, 0)=u_{0}(x), u_{t}(x, 0)=u_{1}(x) \text { in } \Omega
\end{array}\right.
$$

Also, the authors proved the wellposedness by means of nonlinear semigroup method and obtain the uniform stabilization by using the perturbed energy functional method.

Now, we pay attention to our problem. In [11], G. Kirchhoff firstly proposed the so called Kirchhoff string model in the study nonlinear vibration of an elastic string

$$
\rho h u_{t t}+\delta u_{t}=p_{0}+\frac{E h}{2 L}\left(\int_{0}^{L}\left|u_{x}\right|^{2} d x\right) u_{x x}+f, \quad 0<x<L, t>0,
$$

where $u=u(x, t)$ is the lateral displacement at the space coordinate $x$ and the time $t, E$ is the Young modules, $h$ is the cross-section area, $\rho$ is the mass density, $L$ is the length, $p_{0}$ is the initial axial tension, $\delta$ is the resistance modules and $f$ is the external force. More details are present in $[30,31,34,35]$. 
Lots of papers have devoted to the Kirchhoff equation with other boundary conditions. For instance, we can see [18, 19, 21, 22, 23, 30, 31, 34, 35]. It is interesting to observe that problems with condition $M(s)=1$ and a feedback occurs on the boundary were studied by many authors (see $[3,4,5,6,7,12$, $13,14,20])$. It is worth mentioning that M. Aassila [1] has employed Yosida approximation to obtain the global existence for the Kirchhoff equation with nonlinear damping as follows

$$
\left\{\begin{array}{l}
u_{t t}-M\left(\|\nabla u\|^{2}\right) \triangle u+g\left(u_{t}\right)=h(x, t) \text { in } \Omega \times[0, \infty), \\
u(x, t)=0 \text { on } \Gamma_{1} \times[0, \infty), \\
\frac{\partial u}{\partial \nu}=0 \text { on } \Gamma_{0} \times[0, \infty), \\
u(x, 0)=u^{0}(x), u_{t}(x, 0)=u^{1}(x) \text { in } \Omega
\end{array}\right.
$$

It is worth mentioning that Z. Y. Zhang [34] recently have considered the global existence and asymptotic behavior of solutions to initial boundary problem

$$
\left\{\begin{array}{l}
u_{t t}+\gamma \triangle^{2} u-M\left(\|\nabla u\|^{2}\right) \triangle u+\alpha u_{t}+f(u)=0,(x, t) \in \Omega \times(0, \infty) \\
u(x, t)=0, \quad(x, t) \in \partial \Omega \times(0, \infty) \\
u(x, 0)=u_{0}(x), \quad u_{t}(x, 0)=u_{1}(x)
\end{array}\right.
$$

by Banach contraction mapping principle and modified energy functional method.

However, according to our best knowledge, in the present paper, we have to treat Eq.(1.1) with a nonlinear boundary damping condition $g\left(u_{t}\right)$ and nonlinear term $M\left(\|\nabla u\|^{2}\right)$ and it is not considered in the literature. The proof of the existence is based on the Galerkin approximation. For strong solutions to (1.1), this approximation requires a change of variables to transform (1.1) into an equivalent problem with initial value equals zero. Especially, we overcome some difficulties, that is, the presence of nonlinear terms $M\left(\|\nabla u\|^{2}\right)$ and nonlinear boundary damping $g\left(u_{t}\right)$ bring up serious difficulties when passing to the limit, which overcome combining arguments of compacity and monotonicity.

We organize the paper as follows. In Section 2, we give the notations and state our main results. In Section 3, we prove the existence and uniqueness of strong and weak solutions to the problem (1.1) by Galerkin method.

\section{Notations and main results}

In this section, we give some notations which will be used throughout this paper and will state our main results.

Let

$$
V=\left\{v \in H^{1}(\Omega) ; v=0 \text { on } \Gamma_{1}\right\}
$$

and we define

$$
\begin{aligned}
(u, v) & =\int_{\Omega} u(x) v(x) d x,(u, v)_{\Gamma_{0}}=\int_{\Gamma_{0}} u(x) v(x) d x \\
\|u\|^{2} & =\int_{\Omega} u^{2} d x,|u|_{\Gamma_{0}}^{2}=\int_{\Gamma_{0}} u^{2} d x
\end{aligned}
$$


Now, we state the general assumptions:

(A1) Assumptions on $f$ :

Let $f: R \rightarrow R$ be a $W_{l o c}^{1, \infty}(R)$, piecewise $C^{1}(R)$ function,

$$
f(s) s \geq 0 \text { for } s \in R .
$$

Assume that there exists $C_{1}>0$ such that

$$
\left|f^{\prime}(s)\right| \leq C_{1}\left(1+|s|^{p-1}\right), 1<p \leq \frac{n}{n-2} \text { for } s \in R .
$$

Setting $F(s)=\int_{0}^{s} f(\lambda) d \lambda$, there exist $C_{2}, C_{3}>0$ satisfying

$$
C_{2}|s|^{p+1} \leq F(s) \leq C_{3} s f(s) \text { for } s \in R
$$

We notice that from (2.2), we derive that there exists $C_{4}>0$ such that

$$
|f(s)| \leq C_{4}\left(1+|s|^{p}\right) \text { for } s \in R .
$$

Also, we assume that there exists $C_{4}>0$ such that

$$
|f(x)-f(y)| \leq C_{5}\left(|x|^{p-1}+|y|^{p-1}\right)|x-y| \text { for } x, y \in R \text {. }
$$

\section{(A2) Assumptions on $\boldsymbol{g}$ :}

Let $g: R \rightarrow R$ be non-decrease $C^{1}(R)$ function,

$$
g(s) s \geq 0 \text { for } s \neq 0 .
$$

There exist $C_{i}(i=6,7,8,9)$, such that

$$
\begin{gathered}
C_{6}|s| \leq|g(s)| \leq C_{7}|s| \text { if }|s| \leq 1 \\
C_{8}|s|^{q} \leq|g(s)| \leq C_{9}|s|^{q}, 1<q \leq \frac{n-1}{n-2} \text { if }|s|>1 .
\end{gathered}
$$

(A3) Assumptions on $M(s)$ :

Let $M: R \rightarrow R$ be a $C^{1}(R)$ function. Assume that there exist $C_{10}, C_{11}>0$ such that

$$
0<C_{10} \leq M(s) \leq C_{11} \text { for } s>0
$$

Also, we define

$$
\overline{M(s)}=\int_{0}^{s} M(s) d s .
$$

Furthermore, $\overline{M(s)}$ satisfies the following condition

$$
|\overline{M(s)}| \leq s M(s) \text {. }
$$

(A4) Assume that

$$
\left\{u^{0}, u^{1}\right\} \in D(A) \times D(A),
$$

satisfying the compatibility condition

$$
\frac{\partial u}{\partial \nu}+g\left(u_{t}\right)=0 \quad \text { on } \Gamma_{0},
$$

where $D(A)=\{u \in V, \triangle u \in H\}$. 
Notations. In this paper $C$, and $C_{i}$ will denote various positive constants which may be different from line to line and we denote $L^{2}(\Omega)=H$.

Now, we are position to state our main results.

Theorem 2.1. Under assumption (A1)-(A4), the problem (1.1) admits a unique strong solution, that is, a function $u(x, t):[0, \infty) \times \Omega \rightarrow R$, such that

$$
u \in L^{\infty}[0, \infty ; V), u_{t} \in L^{\infty}[0, \infty ; V), u_{t t} \in L^{\infty}\left[0, \infty ; L^{2}(\Omega)\right) .
$$

Theorem 2.2. Assume that $\left\{u^{0}, u^{1}\right\} \in V \times L^{2}(\Omega)$ and assumptions (A1)-(A3) hold. Then the problem (1.1) has a unique weak solutions, $u(x, t):[0, \infty) \times \Omega \rightarrow$ $R$, in the class

$$
u \in C[0, \infty ; V) \cap C^{1}\left[0, \infty ; L^{2}(\Omega)\right)
$$

\section{Existence and uniqueness of strong and weak solutions}

In this section, we prove the existence and uniqueness of strong solutions of the problem (1.1), when $u^{0}, u^{1}$ are smooth. Firstly, we consider strong solutions by Galerkin approximation and we extend the same result to weak solutions using a density argument.

Now, we consider the variational function of (1.1) as follows

$\left(u_{t t}, w\right)+M\left(\|\nabla u\|^{2}\right)(\nabla u, \nabla w)+\alpha\left(u_{t}, w\right)+\left(g\left(u_{t}\right), w\right)_{\Gamma_{0}}+(f(u), w)=0, \forall w \in V$.

Strong solutions to $(1.1)$ with boundary condition $\left(g\left(u_{t}\right), w\right)_{\Gamma_{0}}$ can not be obtained by the method of "special basis", hence, basis formed by eigenfunctions of the operator $-\triangle$ can not be used for it. This leads us to differentiate the variational formulation related with (1.1) with respect to time $t$. But, this brings up serious difficulties when we shall estimate $u_{t t}(0)$.

In order to overcome this difficulties, we can transform the boundary value problem (1.1) into an equivalent one with zero initial data. In fact, we introduce the new variables

$$
v(x, t)=u(x, t)-\phi(x, t)
$$

where

$$
\phi(x, t)=u^{0}(x)+t u^{1}(x), \quad t \in[0, T] .
$$

Due to $(2.11),(2.12),(3.1)-(3.3)$, we get the equivalent problem for variables $v$ :

$$
\left\{\begin{array}{l}
v_{t t}-M\left(\|\nabla v+\nabla \phi\|^{2}\right) \Delta v+\alpha v_{t}+f(v+\phi)=\mathcal{F} \text { in } \Omega \times[0, \infty) \\
v=0 \text { on } \Gamma_{1} \times[0, \infty) \\
\frac{\partial v}{\partial \nu}+g\left(v_{t}+\phi_{t}\right)=\mathcal{G} \text { on } \Gamma_{0} \times[0, \infty) \\
v(0)=v_{t}(0)=0 \text { in } \Omega
\end{array}\right.
$$

where

$$
\mathcal{F}=\Delta \phi+\alpha \phi_{t}, \mathcal{G}=-\frac{\partial \phi}{\partial \nu}
$$


We note that if $v$ is a solution of $(3.4)$ in $[0, T]$, then $u=v+\phi$ is a solution of (1.1) in the same interval.

Hence, using standard methods, we can extend the solution $u$ to the interval $[0, \infty)$. It is sufficient to prove that (3.4) has a local solution by using Galerkin method.

Let $\left(\omega_{j}\right)_{j \in N}$ be basis in $D(A)=V \cap H^{2}(\Omega)$ which is orthonormal in $H$ and setting $V_{m}=\operatorname{span}\left\{\omega_{1}, \omega_{2}, \ldots, \omega_{m}\right\}$.

Now, we define $v_{m}(t)=\sum_{j=1}^{n} g_{j m}(t) \omega_{j}$, where $v_{m}(t)$ is the solution the Cauchy problem as follows:

$$
\begin{aligned}
& \left(v_{m}^{\prime \prime}, w\right)+M\left(\|\nabla v+\nabla \phi\|^{2}\right)\left(\nabla v_{m}, \nabla w\right)+\left(\alpha v_{m}^{\prime}, w\right)+\left(g\left(v_{m}^{\prime}+\phi^{\prime}\right), w\right)_{\Gamma_{0}} \\
& +\left(f\left(v_{m}+\phi\right), w\right)=(\mathcal{F}, w)+(\mathcal{G}, w)_{\Gamma_{0}}, \forall w \in V_{m} \\
& v_{m}(0)=v_{m}^{\prime}(0)=0
\end{aligned}
$$

By standard methods of differential equations, we prove the existence of a solution $(3.6)$ on some interval $\left[0, t_{m}\right]$, then, this solution can be extended to the whole interval $[0, T]$ by use the first estimate as follows.

First Estimate Taking $w=v_{m}^{\prime}$ in (3.6), we obtain

$$
\begin{aligned}
& \frac{1}{2} \frac{d}{d t}\left\{\left\|v_{m}^{\prime}\right\|^{2}+\overline{M\left(\|\nabla v+\nabla \phi\|^{2}\right)}+2 \int_{\Omega} F\left(v_{m}+\phi\right) d x\right\}+\frac{1}{2} \alpha\left\|v_{m}^{\prime}\right\|^{2} \\
& +\left(g\left(v_{m}^{\prime}+\phi^{\prime}\right), v_{m}^{\prime}+\phi^{\prime}\right)_{\Gamma_{0}}=\left(\mathcal{F}, v_{m}^{\prime}\right)+\frac{d}{d t}\left(\mathcal{G}, v_{m}\right)_{\Gamma_{0}}-\left(\mathcal{G}^{\prime}, v_{m}\right)_{\Gamma_{0}} \\
& +\left(f\left(v_{m}+\phi\right), v_{m}^{\prime}\right)+\left(g\left(v_{m}^{\prime}+\phi^{\prime}\right), \phi^{\prime}\right)_{\Gamma_{0}}-M\left(\|\nabla v+\nabla \phi\|^{2}\right)\left(\nabla v_{m}, \nabla u^{1}\right) \\
& -M\left(\|\nabla v+\nabla \phi\|^{2}\right)\left(\nabla v_{m}^{\prime}, \nabla \phi\right)-M\left(\|\nabla v+\nabla \phi\|^{2}\right)\left(\nabla \phi, \nabla \phi^{\prime}\right),
\end{aligned}
$$

i.e.,

$$
\begin{aligned}
& \frac{1}{2} \frac{d}{d t}\left\{\left\|v_{m}^{\prime}\right\|^{2}+\overline{M\left(\|\nabla v+\nabla \phi\|^{2}\right)}+2 \int_{\Omega} F\left(v_{m}+\phi\right) d x\right\} \\
& +\left(g\left(v_{m}^{\prime}+\phi^{\prime}\right), v_{m}^{\prime}+\phi^{\prime}\right)_{\Gamma_{0}} \\
\leq & \left(\mathcal{F}, v_{m}^{\prime}\right)+\frac{d}{d t} I_{1}(t)+I_{2}(t)+I_{3}(t)+I_{4}(t)+I_{5}(t)+I_{6}(t)+I_{7}(t) .
\end{aligned}
$$

Next, we shall estimate for $I_{i}(t)(i=1,2, \ldots, 7)$ in $(3.7)$, respectively.

Due to (2.4) and applying Young's inequality, we get

$$
\begin{aligned}
\left|I_{3}\right| & =\left|\left(f\left(v_{m}+\phi\right), v_{m}^{\prime}\right)\right| \\
& \leq C \int_{\Omega}\left(1+\left|v_{m}+\phi\right|^{p}\right)\left|\phi^{\prime}\right| d x \\
& \leq C\left\{\int_{\Omega}\left|\phi^{\prime}\right| d x+\int_{\Omega}\left|v_{m}+\phi\right|^{p+1} d x+\int_{\Omega}\left|\phi^{\prime}\right|^{p+1} d x\right\} \\
& \leq C+C \int_{\Omega}\left|v_{m}+\phi\right|^{p+1} d x .
\end{aligned}
$$


At the same time, due to $\frac{q}{q+1}+\frac{1}{q+1}=1$, we obtain

$$
\begin{aligned}
\left|I_{4}\right| & =\left|\left(g\left(v_{m}^{\prime}+\phi^{\prime}\right), \phi^{\prime}\right)\right| \\
& \leq \varepsilon \int_{\Gamma_{0}} g\left(v_{m}^{\prime}+\phi^{\prime}\right)^{\frac{q+1}{q}} d \Gamma+C(\varepsilon) \int_{\Gamma_{0}}\left|\phi^{\prime}\right|^{q+1} d \Gamma,
\end{aligned}
$$

where $\varepsilon$ is an arbitrary positive constant.

Also, from (2.7), we deduce

$$
|g(s)|^{\frac{q+1}{q}}=|g(s)||g(s)|^{\frac{1}{q}} \leq C_{11}|g(s)||s|,|s|>1 .
$$

Owing to Poincaré inequality, we conclude

$$
\|v\|_{\Gamma_{0}} \leq C\|\nabla v\|, \forall v \in V
$$

From Cauchy-Schwarz's inequality, we have

$$
\left|I_{2}\right|=\left|\left(\mathcal{G}^{\prime}, v_{m}\right)_{\Gamma_{0}}\right| \leq C\left\{\left\|\mathcal{G}^{\prime}\right\|_{\Gamma_{0}}^{2}+\left\|\nabla v_{m}\right\|^{2}\right\} .
$$

Combining (3.7)-(3.12), it follows that

$$
\begin{aligned}
& \quad \frac{1}{2} \frac{d}{d t}\left\{\left\|v_{m}^{\prime}\right\|^{2}+\overline{M\left(\|\nabla v+\nabla \phi\|^{2}\right)}+2 \int_{\Omega} F\left(v_{m}+\phi\right) d x\right\} \\
& +(1-\varepsilon) \int_{\left|v_{m}^{\prime}+\phi^{\prime}\right|>1}\left|g\left(v_{m}^{\prime}+\phi^{\prime}\right)\right|^{\frac{q+1}{q}} d \Gamma \\
& \leq C(\varepsilon)+\|\mathcal{F}\|^{2}+\left\|\mathcal{G}^{\prime}\right\|_{\Gamma_{0}}^{2}+\frac{d}{d t} I_{1}(t) \\
& \quad+C\left\{\int_{\Omega}\left|v_{m}+\phi\right|^{p+1} d x+\left\|v_{m}^{\prime}\right\|^{2}+\left\|\nabla v_{m}\right\|^{2}\right\}+I_{5}(t)+I_{6}(t)+I_{7}(t) .
\end{aligned}
$$

Now we pay attention to estimating the terms $I_{1}(t), I_{5}(t), I_{6}(t), I_{7}(t)$ in (3.13) as follows.

Due to (3.11), we have

$$
\left|I_{1}\right|=\left|\left(\mathcal{G}, v_{m}\right)_{\Gamma_{0}}\right| \leq \frac{C^{2}}{4 \varepsilon}\|\mathcal{G}\|_{\Gamma_{0}}^{2}+\varepsilon\left\|\nabla v_{m}\right\|^{2} .
$$

From (A3) and Young's inequality,we get

$$
\begin{gathered}
\left|I_{5}\right| \leq C\left\|\nabla v_{m}\right\|^{2}+C, \\
\left|I_{6}\right| \leq C\left\|\nabla v_{m}^{\prime}\right\|^{2}+C, \\
\left|I_{7}\right| \leq C .
\end{gathered}
$$

From (3.13)-(3.17), choosing $\varepsilon>0$ small enough and using Gronwall's inequality and integrating $(3.13)$ over $(0, t)$, observing that $v_{m}(0)=v_{m}^{\prime}(0)=0$, and taking (2.3) into account, it follows that

$$
\left\|v_{m}^{\prime}\right\|^{2}+\overline{M\left(\|\nabla v+\nabla \phi\|^{2}\right)}+\int_{\Omega} F\left(v_{m}+\phi\right) d x+\int_{0}^{t} \int_{\Gamma_{0}}\left|g\left(v_{m}^{\prime}+\phi^{\prime}\right)\right|^{\frac{q+1}{q}} d \Gamma d s \leq C .
$$


Second Estimate Taking $w=v_{m}^{\prime \prime}(0)$ in (3.6) and noticing that $v_{m}(0)=$ $v_{m}^{\prime}(0)=0$, we obtain

$$
\begin{aligned}
& \left\|v_{m}^{\prime \prime}(0)\right\|^{2}+\left(g\left(u^{1}\right), v_{m}^{\prime \prime}(0)\right)_{\Gamma_{0}}+\left(f\left(u^{0}\right), v_{m}^{\prime \prime}(0)\right) \\
= & \left(\overline{M\left(\left\|u^{0}\right\|^{2}\right)} \triangle u^{0}-\alpha \phi_{t}, v_{m}^{\prime \prime}(0)\right)+\left(-\frac{\partial u^{1}}{\partial \nu}, v_{m}^{\prime \prime}(0)\right)_{\Gamma_{0}} .
\end{aligned}
$$

From (A3), (2.12) and (3.19), we obtain

$$
\left\|v_{m}^{\prime \prime}(0)\right\|^{2} \leq\left(\left\|f\left(u^{0}\right)\right\|+\left\|\triangle u^{0}(0)\right\|\right)\left\|v_{m}^{\prime \prime}(0)\right\| .
$$

Owing to (A4), we deduce that

$$
\left\|v_{m}^{\prime \prime}(0)\right\| \leq C .
$$

Also, taking the derivative of (3.6) with respect to time $t$ and taking $w=v_{m}^{\prime \prime}(t)$, we get

$$
\begin{aligned}
& \frac{d}{d t}\left\{\frac{1}{2}\left\|v_{m}^{\prime \prime}\right\|^{2}+\overline{M\left(\|\nabla v+\nabla \phi\|^{2}\right)}\right\}+\int_{\Gamma_{0}} g\left(v_{m}^{\prime}+\phi^{\prime}\right)\left(v_{m}^{\prime \prime}\right)^{2} d \Gamma \\
& +\int_{\Omega} f^{\prime}\left(v_{m}+\phi\right)\left(v_{m}^{\prime}+\phi^{\prime}\right) v_{m}^{\prime \prime} d \Gamma \leq\left(\mathcal{F}^{\prime}, v_{m}^{\prime \prime}\right)+\frac{d}{d t}\left(\mathcal{G}^{\prime}, v_{m}^{\prime}\right)_{\Gamma_{0}} .
\end{aligned}
$$

Next, we shall estimate some terms of $(3.21)$.

Firstly, we estimate $I_{8}=\int_{\Omega} f^{\prime}\left(v_{m}+\phi\right)\left(v_{m}^{\prime}+\phi^{\prime}\right) v_{m}^{\prime \prime} d \Gamma$.

Owing to (2.2), we get

$$
\left|I_{8}\right| \leq C \int_{\Omega}\left(1+\left|v_{m}+\phi\right|^{p-1}\right)\left|v_{m}^{\prime}+\phi^{\prime}\right|\left|v_{m}^{\prime \prime}\right| d x .
$$

Noticing that $\frac{p-1}{2 p}+\frac{1}{2 p}+\frac{1}{2}=1$, from (3.21) and applying the generalized Hölder's inequality, we obtain

$$
\left|I_{8}\right| \leq C\left(\left\|v_{m}^{\prime}+\phi^{\prime}\right\|^{2}+\left\|v_{m}^{\prime \prime}\right\|^{2}\right)+C\left(\left\|v_{m}+\phi\right\|_{\frac{2 p}{p-1}}^{p-1}\left\|v_{m}^{\prime}+\phi^{\prime}\right\|_{2 p}\left\|v_{m}^{\prime \prime}\right\|\right) .
$$

Since $p \leq \frac{n}{n-2}$ and Sobolev embedding theory (see [2]), we get

$$
H_{0}^{1}(\Omega) \hookrightarrow L^{2 p}(\Omega) .
$$

From (3.23) and (3.24), we obtain that

$$
\left|I_{8}\right| \leq C\left(1+\left\|v_{m}^{\prime \prime}\right\|^{2}+\left\|\nabla v_{m}^{\prime}\right\|^{2}\right) .
$$

Secondly, we estimate $I_{9}=\int_{\Gamma_{0}} g\left(v_{m}^{\prime}+\phi^{\prime}\right)\left(v_{m}^{\prime \prime}\right)^{2} d \Gamma$.

Noticing that $g^{\prime}(s) \geq 0$, we deduce that $I_{9} \geq 0$.

Combining (3.21), (3.23) and (3.25), we get

$$
\begin{aligned}
& \frac{d}{d t}\left\{\frac{1}{2}\left\|v_{m}^{\prime \prime}\right\|^{2}+\overline{M\left(\|\nabla v+\nabla \phi\|^{2}\right)}\right\} \\
\leq & \left\|\mathcal{F}^{\prime}\right\|^{2}+\frac{d}{d t}\left(\mathcal{G}^{\prime}, v_{m}^{\prime}\right)_{\Gamma_{0}}+C\left(1+\left\|v_{m}^{\prime \prime}\right\|^{2}+\left\|\nabla v_{m}^{\prime}\right\|^{2}\right) .
\end{aligned}
$$


Integrating (3.26) over the interval $(0, t)$, and noticing that $(3.20)$ and $v_{m}^{\prime}(0)=$ 0 , it follows that

$$
\left\|v_{m}^{\prime \prime}\right\|^{2}+\overline{M\left(\|\nabla v+\nabla \phi\|^{2}\right)} \leq C+\left(\mathcal{G}^{\prime}, v_{m}^{\prime}\right)_{\Gamma_{0}}+C \int_{0}^{t}\left(\left\|v_{m}^{\prime \prime}\right\|^{2}+\left\|\nabla v_{m}^{\prime}\right\|^{2}\right) d s .
$$

Also, by Young's inequality, we have

$$
\left(\mathcal{G}^{\prime}, v_{m}^{\prime}\right)_{\Gamma_{0}} \leq \frac{C^{2}}{4 \varepsilon}\left\|\mathcal{G}^{\prime}\right\|_{\Gamma_{0}}^{2}+\varepsilon\left\|\nabla v_{m}^{\prime}\right\|^{2} .
$$

From (3.27), (3.28) and (A3), choosing $\varepsilon>0$ sufficiently small enough and applying Gronwall's inequality, we get

$$
\left\|v_{m}^{\prime \prime}\right\|^{2}+\left\|\nabla v_{m}^{\prime}\right\|^{2} \leq C .
$$

Next, we shall prove existence of solutions and analyze the nonlinear terms $f, g, h$.

\section{Analysis of $f$ :}

From (2.4) and the First Estimate, we have

$$
\int_{0}^{t} \int_{\Omega} f\left(v_{m}+\phi\right)^{\frac{p+1}{p}} d x d s \leq C \int_{0}^{t} \int_{\Omega}\left(1+\left|v_{m}+\phi\right|^{\frac{p+1}{p}}\right) d x d s \leq C .
$$

The inequality (3.30) implies that

$$
\left\{f\left(v_{m}+\phi\right)\right\} \text { is bounded in } L^{\frac{p+1}{p}}\left(Q_{T}\right),
$$

where $Q_{T}=\Omega \times(0, T)$.

From (3.29), we can find a subsequence $v_{m}$ (still denote $v_{m}$ ) such that

$$
v_{m} \rightarrow v \text { strong in } L^{2}\left(Q_{T}\right),
$$

then, $v_{m} \rightarrow v$ a.e in $Q_{T}$. Hence, we get

$$
f\left(v_{m}+\phi\right) \rightarrow f(v+\phi) \text { a.e in } Q_{T} .
$$

Due to (3.31), (3.33) and applying Lions lemma (see [16]), we deduce

$$
f\left(v_{m}+\phi\right) \rightarrow f(v+\phi) \text { weakly in } L^{\frac{p+1}{p}}\left(Q_{T}\right) .
$$

\section{Analysis of $g$ :}

From the First Estimate, we obtain

$$
\left\{g\left(v_{m}^{\prime}+\phi^{\prime}\right)\right\} \text { is bounded in } L^{\frac{q+1}{q}}(\Sigma),
$$

where $\Sigma=\Gamma_{0} \times(0, T)$. So, there exists $\varrho \in L^{\frac{q+1}{q}}(\Sigma)$, such that

$$
g\left(v_{m}^{\prime}+\phi^{\prime}\right) \rightarrow \varrho \text { weakly in } L^{\frac{q+1}{q}}(\Sigma) .
$$

From the First and Second Estimate, and Sobolev embedding theory, it is easy to see that

$$
v_{m}^{\prime} \rightarrow v \text { in } L^{2}\left(0, \infty, L^{2}(\Omega)\right)
$$


We also notice that

$$
\begin{aligned}
\int_{\Gamma_{0}}\left|g\left(v_{m}^{\prime}+\phi^{\prime}\right)\right|^{2} d \Gamma & =\int_{\left|v_{m}^{\prime}+\phi^{\prime}\right| \leq 1}\left|g\left(v_{m}^{\prime}+\phi^{\prime}\right)\right|^{2} d \Gamma+\int_{\left|v_{m}^{\prime}+\phi^{\prime}\right|>1}\left|g\left(v_{m}^{\prime}+\phi^{\prime}\right)\right|^{2} d \Gamma \\
& \leq C+C\left\|v_{m}^{\prime}+\phi^{\prime}\right\|_{2 q, \Gamma_{0}}^{2 q} \\
& \leq C+C\left\|\nabla v_{m}^{\prime}+\nabla \phi^{\prime}\right\|^{2 q} \\
& \leq C .
\end{aligned}
$$

Then, we deduce that

$$
g\left(v_{m}^{\prime}+\phi^{\prime}\right) \rightarrow \varrho \text { weakly in } L^{2}(\Sigma) .
$$

Analysis of $M\left(\|\nabla u\|^{2}\right)$ :

From now on we are interested in the convergence of the nonlinear term. We define

$$
\psi_{m}(t)=\left\|\nabla v_{m}(t)\right\|^{2}, \quad t \in[0, T] .
$$

From the second estimate we obtain

$$
\left|\psi_{m}(t)\right| \leq C, \quad \forall m \in N, \quad t \in[0, T] .
$$

Now, if $t_{1}, t_{2} \in[0, T]$, we can get

$$
\left|\psi_{m}\left(t_{1}\right)-\psi_{m}\left(t_{2}\right)\right| \leq \int_{t_{1}}^{t_{2}}\left|\psi_{m}^{\prime}(s)\right| d s .
$$

On the other hand, from the above estimates we deduce

$$
\psi_{m}^{\prime}(s)=2\left(\nabla v_{m}(s), \nabla v_{m}^{\prime}(s)\right) \leq C .
$$

From (3.40) and (3.41), it follows that

$$
\left|\psi_{m}\left(t_{1}\right)-\psi_{m}\left(t_{2}\right)\right| \leq C\left|t_{1}-t_{2}\right| .
$$

Then, owing to (3.39) and (3.42) by Arezela-Ascoli's theorem [29], there exists a continuous function $\psi:[0, T] \rightarrow R$ such that

$$
\psi_{m}(t) \rightarrow \psi(t)
$$

uniformly in $[0, T]$ and, since (A3), we have

$$
M\left(\left|\psi_{m}(t)\right|\right) \rightarrow M(|\psi(t)|)
$$

uniformly in $[0, T]$.

Also, we note that from the first and second estimates and noticing that $\|v\|_{H^{\frac{1}{2}\left(\Gamma_{0}\right)}} \leq C|\nabla v|$ for all $v \in V$, we get

$$
\begin{aligned}
& \left\{v_{m}\right\} \text { is bounded in } L^{2}\left(0, T ; H^{\frac{1}{2}}\left(\Gamma_{0}\right)\right), \\
& \left\{v_{m}^{\prime}\right\} \text { is bounded in } L^{2}\left(0, T ; L^{2}\left(\Gamma_{0}\right)\right) .
\end{aligned}
$$


Because of the injection $H^{\frac{1}{2}}\left(\Gamma_{0}\right) \hookrightarrow L^{2}\left(\Gamma_{0}\right)$ is continuous and compact, making use of Aubin-Lions theorem [17], there exists a subsequence of $v_{m}$ (still denote $v_{m}$ ) such that

$$
v_{m} \rightarrow v \text { strongly in } L^{2}\left(0, T ; L^{2}\left(\Gamma_{0}\right)\right) .
$$

From Analysis of $f$ and above convergence, we get

$$
v_{m}^{\prime \prime} \rightarrow v^{\prime \prime} \text { weakly in } L^{2}\left(0, T, L^{2}(\Omega)\right) \text {. }
$$

So, we can pass to the limit in the approximate system given by (3.6) using standard arguments in order to obtain

$$
u_{t t}-M\left(\|\nabla u\|^{2}\right) \triangle u+\alpha u_{t}+f(u)=0 \text { in } L^{2}\left(0, T, L^{2}(\Omega)\right) .
$$

Also, we have

$$
\frac{\partial v}{\partial \nu}+\varrho=\mathcal{G} \text { in } L^{2}\left(0, \infty, L^{2}\left(\Gamma_{0}\right)\right) .
$$

Indeed, we consider $w=v_{m}$ in (3.6) and integrate over $(0, T)$, then we get

$$
\begin{aligned}
& \int_{0}^{T}\left(v_{m}^{\prime \prime}, v_{m}\right) d t+\int_{0}^{T} M\left(\|\nabla v+\nabla \phi\|^{2}\right)\left|\nabla v_{m}\right|^{2} d t+\alpha \int_{0}^{T}\left(v_{m}^{\prime}, v_{m}\right) d t \\
& +\int_{0}^{T}\left(f\left(v_{m}+\phi\right), v_{m}\right) d t \\
= & \int_{0}^{T}\left(\mathcal{F}, v_{m}\right) d t+\int_{0}^{T}\left(\mathcal{G}, v_{m}\right)_{\Gamma_{0}} d t .
\end{aligned}
$$

Combining above estimate and convergence, we deduce that

$$
v_{m}^{\prime \prime} \rightarrow v^{\prime \prime} \text { weakly in } L^{2}\left(0, T, L^{2}(\Omega)\right) .
$$

Hence, we can pass to the limit in (3.50) to deduce that

$$
v^{\prime \prime}-M\left(\|\nabla v+\nabla \phi\|^{2}\right) \triangle v+\alpha v^{\prime}+f(v+\phi)=\mathcal{F} \in L^{2}(0, \infty ; H) .
$$

Next, we shall show $\varrho=g\left(v+\phi^{\prime}\right)$. For this goal, we shall use monotonicity arguments.

First of all, we notice that from the First and Second estimates and applying Aubin-Lions Theorem (see [17]), then we have

$$
v_{m}^{\prime} \rightarrow v^{\prime} \text { weakly in } L^{2}(0, T ; H) .
$$

Considering $w=v_{m}^{\prime}$ in (3.6), integrating over $[0, T]$, we deduce

$$
\begin{gathered}
v^{\prime \prime}-M\left(\|\nabla v+\nabla \phi\|^{2}\right) \triangle v+\alpha v^{\prime}+f(v+\phi)=\mathcal{F} \in L^{2}(0, \infty ; H), \\
\frac{\partial v}{\partial \nu}+\varrho=\mathcal{G} \in L^{2}\left(0, \infty ; L^{2}\left(\Gamma_{0}\right)\right) .
\end{gathered}
$$

Also, we have

$$
\lim _{m \rightarrow \infty} \int_{0}^{T}\left(g\left(v_{m}^{\prime}+\phi^{\prime}\right), v_{m}^{\prime}+\phi^{\prime}\right) d t=\int_{0}^{T}\left(\varrho, v^{\prime}+\phi^{\prime}\right)_{\Gamma_{0}} d t .
$$


Owing to function $g$ is a non-decreasing monotone function, we have

$$
\int_{0}^{T}\left\langle g\left(v_{m}^{\prime}+\phi^{\prime}\right)-g(\psi), v_{m}^{\prime}+\phi^{\prime}-\psi\right\rangle d t \geq 0, \forall \psi \in L^{q+1}\left(\Gamma_{0}\right),
$$

where $\langle\cdot, \cdot\rangle$ means the duality between Sobolev spaces $L^{\frac{q+1}{q}}\left(\Gamma_{0}\right)$ and $L^{q+1}\left(\Gamma_{0}\right)$. So, we deduce

$$
\begin{aligned}
& \int_{0}^{T}\left\langle g\left(v_{m}^{\prime}+\phi^{\prime}\right), \psi\right\rangle d t+\int_{0}^{T}\left\langle g(\psi), v_{m}^{\prime}+\phi^{\prime}-\psi\right\rangle d t \\
\leq & \int_{0}^{T}\left\langle g\left(v_{m}^{\prime}+\phi^{\prime}\right), v_{m}^{\prime}+\phi^{\prime}\right\rangle d t .
\end{aligned}
$$

From (3.54), we get

$$
\begin{aligned}
& \lim _{m \rightarrow \infty} \inf \int_{0}^{T}\left\langle g\left(v_{m}^{\prime}+\phi^{\prime}\right), \psi\right\rangle d t+\lim _{m \rightarrow \infty} \inf \int_{0}^{T}\left\langle g(\psi), v_{m}^{\prime}+\phi^{\prime}-\psi\right\rangle d t \\
\leq & \lim _{m \rightarrow \infty} \inf \int_{0}^{T}\left\langle g\left(v_{m}^{\prime}+\phi^{\prime}\right), v_{m}^{\prime}+\phi^{\prime}\right\rangle d t .
\end{aligned}
$$

Since

$$
\left\|v_{m}\right\|_{q+1, \Gamma_{0}} \leq C\left\|\nabla v_{m}^{\prime}\right\| \leq C
$$

then, it follows that

$$
v_{m}^{\prime} \rightarrow v^{\prime} \text { weakly star in } L^{\infty}\left(0, T ; L^{q+1}\left(\Gamma_{0}\right)\right) .
$$

From (3.51), (3.53), (3.55) and (3.56), we obtain

$$
\int_{0}^{T}\left\langle\varrho-g(\psi), v_{m}^{\prime}+\phi^{\prime}-\psi\right\rangle d t \geq 0
$$

Finally, we apply the monotone method to obtain $\varrho=g\left(v^{\prime}+\phi^{\prime}\right)$.

Setting $\psi=\left(v^{\prime}+\phi^{\prime}\right)+\lambda \xi$ in (3.57), where $\xi$ is an arbitrary element of $L^{q+1}\left(\Gamma_{0}\right)$ and $\lambda>0$, we obtain

$$
\int_{0}^{T}\left\langle\varrho-g\left(v^{\prime}+\phi^{\prime}+\lambda \xi\right),-\lambda \xi\right\rangle d t \geq 0
$$

So,

$$
\int_{0}^{T}\left\langle\varrho-g\left(v^{\prime}+\phi^{\prime}+\lambda \xi\right),-\xi\right\rangle d t \leq 0, \forall \xi \in L^{q+1}\left(\Gamma_{0}\right) .
$$

As for the operator

$$
g: L^{q+1}\left(\Gamma_{0}\right) \rightarrow L^{\frac{q+1}{q}}\left(\Gamma_{0}\right): v \mid \rightarrow g(v)
$$

is hemi-continuous and we get

$$
\int_{0}^{T}\left\langle\varrho-g\left(v^{\prime}+\phi^{\prime}\right), \xi\right\rangle d t \leq 0, \forall \xi \in L^{q+1}\left(\Gamma_{0}\right)
$$


Also, we have

$$
\int_{0}^{T}\left\langle\varrho-g\left(v^{\prime}+\phi^{\prime}\right), \xi\right\rangle d t \geq 0, \forall \xi \in L^{q+1}\left(\Gamma_{0}\right) .
$$

Hence,

$$
\int_{0}^{T}\left\langle\varrho-g\left(v^{\prime}+\phi^{\prime}\right), \xi\right\rangle d t=0, \forall \xi \in L^{q+1}\left(\Gamma_{0}\right)
$$

which implies

$$
\varrho=g\left(v^{\prime}+\phi^{\prime}\right)
$$

\section{Uniqueness:}

Let $u_{1}$ and $u_{2}$ be two smooth solutions to problem (1.1), then, $z=u_{1}-u_{2}$ satisfies

$$
\begin{aligned}
& \left(z^{\prime \prime}, w\right)+M\left(\left\|\nabla u_{2}\right\|^{2}\right)(\nabla z, \nabla w)+\left(M\left(\left\|\nabla u_{2}\right\|^{2}\right)-M\left(\left\|\nabla u_{1}\right\|^{2}\right)\right)\left(\nabla u_{1}, \nabla w\right) \\
& +\left(\alpha z^{\prime}, w\right)+\left(g\left(u_{1}^{\prime}\right)-g\left(u_{2}^{\prime}\right), w\right)_{\Gamma_{0}}=\left(f\left(u_{2}\right)-f\left(u_{1}\right), w\right), \forall w \in V, \\
& z(0)=z^{\prime}(0)=0 .
\end{aligned}
$$

Putting $w=z^{\prime}(t)$ in (3.59), we deduce

$$
\begin{aligned}
& \frac{1}{2} \frac{d}{d t}\left\{\left\|z^{\prime}\right\|^{2}+M\left(\left\|\nabla u_{2}\right\|^{2}\right)\left(\nabla z, \nabla z^{\prime}\right)+\left(\alpha z^{\prime}, z^{\prime}\right)+\left(g\left(u_{1}^{\prime}\right)-g\left(u_{2}^{\prime}\right), z^{\prime}\right)_{\Gamma_{0}}\right. \\
= & \left(f\left(u_{2}\right)-f\left(u_{1}\right), w\right)+\left(M\left(\left\|\nabla u_{1}\right\|^{2}\right)-M\left(\left\|\nabla u_{2}\right\|^{2}\right)\right)\left(\nabla u_{1}, \nabla z^{\prime}\right) .
\end{aligned}
$$

Next, we estimate the terms in the right hand side of (3.55).

Estimate for $J_{1}=\left(f\left(u_{2}\right)-f\left(u_{1}\right), z^{\prime}\right)$

From (2.5), we get

$$
\begin{aligned}
\left|J_{1}\right| & \leq C \int_{\Omega}\left(\left|u_{2}\right|^{p-1}+\left|u_{1}\right|^{p-1}\right)\left|z \| z^{\prime}\right| d x \\
& \leq C\left(\left\|u_{2}\right\|_{2 p}+\left\|u_{1}\right\|_{2 p}\right)\|z\|_{2 p}\left\|z^{\prime}\right\| \\
& \leq C\left(\|\nabla z\|^{2}+\left\|z^{\prime}\right\|^{2}\right) .
\end{aligned}
$$

Estimate for $J_{2}=\left|M\left(\left\|\nabla u_{1}\right\|^{2}\right)-M\left(\left\|\nabla u_{2}\right\|^{2}\right)\right|$

Since $M$ is $C^{1}$, we get

$$
\begin{aligned}
\left|J_{2}\right| & \leq C\left|\left\|\nabla u_{1}\right\|^{2}-\left\|\nabla u_{2}\right\|^{2}\right| \\
& \leq C\left(\left\|\nabla u_{1}\right\|+\left\|\nabla u_{2}\right\|\right)\left(\left\|\nabla u_{1}\right\|-\left\|\nabla u_{2}\right\|\right) \\
& \leq C\left(\|\nabla z\|^{2}+\left\|z^{\prime}\right\|^{2}\right) .
\end{aligned}
$$

From (A4), (3.60)-(3.62), observing that $g$ is monotone function and making using of Gronwall's inequality, we deduce $\|\nabla z\|=\left\|z^{\prime}\right\|=0$ and so, $u_{2}=u_{1}$.

Then, we complete the proof of existence and uniqueness of smooth solutions.

\section{Existence of Weak Solutions}


Consider

$$
\left\{u^{0}, u^{1}\right\} \in V \times H
$$

and set

$$
D(-\triangle)=\left\{v \in V \cap H^{2}(\Omega) ; \frac{\partial v}{\partial \nu}=0 \text { on } \Gamma_{0}\right\} .
$$

Due to Sobolev space theory (see [4]), we get

$$
D(-\triangle) \text { is dense in } V \text { and } H_{0}^{1}(\Omega) \cap H^{2}(\Omega) \text { is dense in } H,
$$

then, there exist $\left\{u_{\mu}^{0}\right\} \subset D(-\triangle)$ and $\left\{u_{\mu}^{1}\right\} \subset H_{0}^{1}(\Omega) \cap H^{2}(\Omega)$, such that

$$
\begin{aligned}
& u_{\mu}^{0} \rightarrow u^{0} \text { strongly in } V, \\
& u_{\mu}^{1} \rightarrow u^{1} \text { strongly in } H .
\end{aligned}
$$

Furthermore, $\frac{\partial u^{0}}{\partial \nu}+g\left(u_{\mu}^{1}\right)=0$ on $\Gamma_{0}$. Therefore, there exists $u_{\mu}: Q \rightarrow R$ smooth solution of problem (1.1) satisfying

$$
\left\{\begin{array}{l}
u_{\mu}^{\prime \prime}-M\left(\left\|\nabla u_{\mu}\right\|^{2}\right) \triangle u_{\mu}+\alpha u_{\mu}^{\prime}+f\left(u_{\mu}\right)=0 \text { in } L^{2}([0, \infty ; H), \\
u_{\mu}=0 \text { on } \Gamma_{1}, \\
\frac{\partial u_{\mu}}{\partial \nu}+g\left(u_{\mu}^{\prime}\right)=0 \text { on } L^{2}\left(\left[0, \infty ; L^{2}\left(\Gamma_{0}\right)\right),\right. \\
u_{\mu}(0)=u_{\mu}^{0}, u_{\mu}^{\prime}(0)=u_{\mu}^{1} .
\end{array}\right.
$$

Repeating the same discussions, we get

$$
\left\|u_{\mu}^{\prime}\right\|^{2}+\overline{M\left(\left\|\nabla u_{\mu}\right\|^{2}\right)}+\int_{0}^{t} \int_{\Omega}\left|u_{\mu}\right|^{p+1} d x d t+\int_{0}^{t} \int_{\Gamma_{0}}\left|g\left(u_{\mu}^{\prime}\right)\right|^{\frac{q+1}{q}} d \Gamma d s \leq C
$$

and

$$
\begin{gathered}
\int_{0}^{t} \int_{\Omega}\left|f\left(u_{\mu}\right)\right|^{\frac{p+1}{p}} d x d s \leq C, \\
\int_{0}^{t} \int_{\Gamma_{0}}\left|u_{\mu}^{\prime}\right|^{q+1} d \Gamma d s \leq C,
\end{gathered}
$$

for all $t \in[0, T]$ and $\mu \in N$.

Setting $z_{\mu \sigma}=u_{\mu}-u_{\sigma}, \mu, \sigma \in N$, where $u_{\mu}, u_{\sigma}$ are smooth solutions of (3.66).

Repeating the same discussions used in the existence and uniqueness of strong solutions, we deduce that there exists $u: Q \rightarrow R$ such that

$$
\begin{gathered}
u_{\mu} \rightarrow u \text { in } L^{\infty}([0, T] ; V), \\
u_{\mu}^{\prime} \rightarrow u^{\prime} \text { in } L^{\infty}([0, T] ; H) .
\end{gathered}
$$

Furthermore, from (3.67), (3.70) and (2.6), we get

$$
\begin{gathered}
u_{\mu}^{\prime} \rightarrow u^{\prime} \text { weakly in } L^{q+1}(\Sigma), \\
f\left(u_{\mu}\right) \rightarrow \eta \text { weakly in } L^{\frac{p+1}{p}}\left(Q_{T}\right), \\
g\left(u_{\mu}^{\prime}\right) \rightarrow \chi \text { weakly in } L^{\frac{q+1}{q}}(\Sigma) .
\end{gathered}
$$


From (3.66) and Lion's Lemma (see [17]), it is easy to obtain that $\eta=f(u)$.

Furthermore, we have

$$
\left\{\begin{array}{l}
u_{t t}-M\left(\|\nabla u\|^{2}\right) \triangle u+\alpha u_{t}+f(u)=0 \\
u(x, 0)=u_{0}, \quad u_{t}(x, 0)=u_{1} .
\end{array}\right.
$$

Next, we shall prove $\chi=g\left(u^{\prime}\right)$.

Indeed, multiplying the first equation (3.66) by $u_{\mu}$ and integrating over $\Omega$, we obtain

$$
\begin{aligned}
& \frac{1}{2} \frac{d}{d t}\left\{\left\|u_{\mu}^{\prime}\right\|^{2}+\overline{M\left(\left\|\nabla u_{\mu}\right\|^{2}\right)}\right\}+\alpha\left\|u_{\mu}^{\prime}\right\|^{2} \\
& +\left(f\left(u_{\mu}\right), u_{\mu}^{\prime}\right)+\left(g\left(u_{\mu}^{\prime}\right), u_{\mu}^{\prime}\right)_{\Gamma_{0}}=0 .
\end{aligned}
$$

Also, we have

$$
\begin{aligned}
& \frac{1}{2}\left\|u_{\mu}^{\prime}\right\|^{2}+\frac{1}{2} \overline{M\left(\left\|\nabla u_{\mu}\right\|^{2}\right)}+\alpha \int_{0}^{t}\left\|u_{\mu}^{\prime}\right\|^{2}+\int_{0}^{t}\left(f\left(u_{\mu}(s)\right), u_{\mu}^{\prime}(s)\right) d s \\
& +\int_{0}^{t}\left(g\left(u_{\mu}^{\prime}(s)\right), u_{\mu}^{\prime}(s)\right)_{\Gamma_{0}} d s \\
= & \frac{1}{2}\left\|u_{\mu}^{1}\right\|^{2}+\frac{1}{2}\left\|\nabla u_{\mu}^{0}\right\|^{2}+\frac{1}{2} \overline{M\left(\left\|\nabla u_{\mu}^{0}\right\|^{2}\right)} .
\end{aligned}
$$

From (3.65), (3.66), (3.68), (3.69), (3.71), (3.73) and (3.76), we deduce

$$
\begin{aligned}
& \lim _{\mu \rightarrow \infty} \int_{0}^{t}\left(g\left(u_{\mu}^{\prime}(s)\right), u_{\mu}^{\prime}(s)\right)_{\Gamma_{0}} d s \\
= & \frac{1}{2}\left\|u^{1}\right\|^{2}+\frac{1}{2}\left\|\nabla u^{0}\right\|^{2}-\frac{1}{2}\left\|u^{\prime}\right\|^{2}-\frac{1}{2} \overline{M\left(\|\nabla u\|^{2}\right)}+\alpha\left\|u^{\prime}\right\|^{2} \\
& -\int_{0}^{t}\left(f(u(s)), u^{\prime}(s)\right) d s+\frac{1}{2} \overline{M\left(\left\|\nabla u_{\mu}^{0}\right\|^{2}\right)} .
\end{aligned}
$$

At the same time, assuming that $\omega$ is a weak solution to problem

$$
\left\{\begin{array}{l}
\omega^{\prime \prime}-M\left(\|\nabla \omega\|^{2}\right) \triangle \omega+\alpha \omega^{\prime}+f(\omega)=0 \text { in } \Omega \times(0, \infty) \\
\omega=0 \text { on } \Gamma_{1} \times(0, \infty) \\
\frac{\partial \omega}{\partial \nu}+\chi=0 \text { on } \Gamma_{0} \times(0, \infty), \\
\omega(0)=u^{0}, \omega^{\prime}(0)=u^{1}
\end{array}\right.
$$

then, we have

$$
\begin{aligned}
& \left.\int_{0}^{t}(\chi(s)), \omega^{\prime}(s)\right)_{\Gamma_{0}} d s \\
= & \frac{1}{2}\left\|u^{1}\right\|^{2}+\frac{1}{2}\left\|\nabla u^{0}\right\|^{2}-\frac{1}{2}\left\|\omega^{\prime}\right\|^{2}+\overline{M\left(\|\nabla \omega\|^{2}\right)}-\alpha\left\|\omega^{\prime}\right\|^{2} \\
& -\int_{0}^{t}\left(f(\omega(s)), \omega^{\prime}(s)\right) d s .
\end{aligned}
$$


Since $u$ is a weak solution to problem (3.78), hence, from (3.77) and (3.79), we get

$$
\left.\lim _{\mu \rightarrow \infty} \int_{0}^{t}\left(g\left(u_{\mu}^{\prime}(s)\right), u_{\mu}^{\prime}(s)\right)_{\Gamma_{0}} d s=\int_{0}^{t}(\chi(s)), u^{\prime}(s)\right)_{\Gamma_{0}} d s .
$$

According to the above arguments, it is easy to show that $\chi=g\left(u^{\prime}\right)$.

Remark 3.1. For the uniqueness of weak solutions, we require a regularization procedure using standard arguments (see [17]).

Acknowledgement. The authors are highly grateful for the anonymous referee's valuable comments and suggestions on this note. I would like to express my sincere gratitude to Dr. Professor Jian hua Huang for his enthusiastic concern and constant encouragement. This work was supported by China Postdoctoral Science Foundation No.2013M532169, NSF of Hunan Province Grant No.11JJ3004 and NNSF of China Grant No.11371367.

\section{References}

[1] M. Aassila, Asymptotic behavior of solutions to a quasilinear hyperbolic equation with nonlinear damping, Electron. J. Qual. Theory Differ. Equ. 1998 (1998), no. 7, 12 pp.

[2] R. A. Adams, Sobolev Space, Acadmic Press, New York, 1975.

[3] A. Arosio and S. Spagnolo, Global solutions of the Cauchy problem for a nonlinear hyperbolic equation, Nonlinear Differential Equations and Their Applications, College de France Seminar, 6, Pitman, London, 1984.

[4] M. M. Cavalcanti, V. N. Domings Cavalcanti, J. S. Prates Filho, and J. A. Soriano, Existence and exponential decay for a Kirchhoff-Carrier model with viscosity, J. Math. Anal. Appl. 226 (1998), no. 1, 20-40.

[5] - Existence and uniform decay of solutions of a degenarate equation nonlinear boundary damping and boundary memory source term, Nonlinear Analysis T. M. A. 38 (1999), 281-294.

[6] _ Existence and uniform decay rates for viscoelastic problems with nonlinear boundary damping, Differential Integral Equations 14 (2001), no. 1, 85-116.

[7] M. M. Cavalcanti, V. N. Domings Cavalcanti, and J. A. Soriano, On existence and asymptotic stability of solutions of the degenerate wave equation with nonlinear boundary conditions, J. Math. Anal. Appl. 281 (2003), no. 1, 108-124.

[8] F. Gazzola and M. Squassina, Global solutions and finite time blow up for damped semilinear wave equations, Ann. Inst. H. Poincaré Anal. Non Linéaire 23 (2006), no. 2, 185-207.

[9] R. Ikehata, Some remarks on the wave equations with nonlinear damping and source terms, Nonlinear Anal. 54 (1996), no. 10, 1165-1175.

[10] N. I. Karachalios and N. M. Stavrakakis, Global existence and blow up results for some nonlinear wave equations on $R^{n}$, Adv. Differential Equations 6 (2001), no. 2, 155-174.

[11] G. Kirchhoff, Vorlesungen Uber Mechanik, Teubner, Leipzig, 1883.

[12] J. E. Lagnese, Note on boundary stabilization of wave equations, SIAM J. Control Optim. 26 (1988), no. 5, 1250-1257.

[13] I. Lasiecka and J. Ong, Global sovability and uniform decays of solutions to quasilinear equation with nonlinear boundary conditions, Communications in PDE 24 (1999), 20692109.

[14] I. Lasiecka and D. Tataru, Uniform boundary stabilization of semilinear wave equations with nonlinear boundary damping, Differential Integral Equations 6 (1993), no. 3, 507533 . 
[15] H. A. Levine and S. Park, Global existence and global nonexistence of solutions of the Cauchy problem for a nonlinearly wave equation, J. Math. Anal. Appl. 228 (1998), no. 1, 181-205.

[16] J. L. Lions, Quelques Méthodes Résolution des Problemes aux Limites Non-Lineares, Dunod, Paris, 1969.

[17] J. L. Lions and E. Magenes, Problemes aux limites non homogenes applications, Dunod, Paris, 1, 1968.

[18] T. Matsuyama and R. Ikehata, On global solutions and energy decay for the wave equations of Kirchhoff type with nonlinear damping terms, J. Math. Anal. Appl. 204 (1996), no. 3, 729-753.

[19] K. Narasimha, Nonlinear vibration of an elastic string, J. Sound Vib. 8 (1968), 134-146.

[20] K. Narasimha and Yamada, On global solutions of some degenerate quasilinear hyperbolic equation with dissipative terms, Funkcialaj Ekvacioj 33 (1990), 151-159.

[21] K. Ono, On global existence, asymtotic stability and blow-up of solutions for some degenerate nonlinear wave equations of Kirchhoff type, Math. Methods. Appl. Sci. 20 (1997), 151-177.

[22] - On global solutions and blow-up of solutions of nonlinear Kirchhoff string with nonlinear dissipation, J. Math. Anal. Appl. 216 (1997), 321-342.

[23] - Global existence, decay, and blowup of solutions for some mildly degenerate nonlinear Kirchhoff strings, J. Differential Equations 137 (1997), no. 2, 273-301.

[24] J. Y. Park and J. J. Bae, On the existence of solutions of strongly damped wave equations, Internat. J. Math. and Math. Sci. 23 (2000), no. 6, 369-382.

[25] R. Pitts and M. A. Rammaha, Global existence and non-existence theorems for nonlinear wave equations, Indiana Univ. Math. J. 51 (2002), no. 6, 1479-1509.

[26] M. A. Rammaha and T. A. Strei, Global existence and nonexistence for nonlinear wave equations with damping and source terms, Trans. Amer. Math. Soc. 354 (2002), no. 9, 3621-3637 (electrolic).

[27] I. Segal, Nonlinear semigroups, Ann. of Math. 78 (1963), 339-364.

[28] Z. J. Yang, Initial boundary value problem for a class of non-linear strongly damped wave equations, Math. Methods. Appl. Sci. 26 (2003), no. 12, 1047-1066.

[29] K. Yosida, Fuctional Analysis, Sringer-Verlag, New York, 1996.

[30] Z. Y. Zhang, Central manifold for the elastic string with dissipative effect, Pacific Journal of Applied Mathematics 4 (2010), no. 2, 329-343.

[31] Z. Y. Zhang, Z. H. Liu, and X. J. Miao, Estimate on the dimension of global attractor for nonlinear dissipative Kirchhoff equation, Acta Appl. Math. 110 (2010), no. 1, 271-282.

[32] Z. Y. Zhang, Z. H. Liu, X. J. Miao, and Y. Z. Chen, Global existence and uniform stabilization of a generalized dissipative Klein-Gordon equation type with boundary damping, Journal of Mathematics and Physics 52 (2011), no. 2, 023502, 12 pp.

[33] Z. Y. Zhang and X. J. Miao, Global existence and uniform decay for wave equation with dissipative term and boundary damping, Comput. Math. Appl. 59 (2010), no. 2, 1003-1018.

[34] _ Existence and asymptotic behavior of solutions to generalized Kirchhoff equation, Nonlinear Stud. 19 (2012), no. 1, 57-70.

[35] Z. Y. Zhang, X. J. Miao, and D. M. Yu, On solvability and stabilization of a class of hyperbolic hemivariational inequalities in elasticity, Funkcial. Ekvac. 54 (2011), no. 2, $297-314$. 
ZAI-YUN ZHANG

College of SCIENCE

National University of Defense Technology

Changsha, 410083, Hunan, P. R. China

AND

School of Mathematics

Hunan Institute of Science and Technology

Yueyang, 414006, Hunan, P. R. China

E-mail address: zhangzaiyun1226@126.com

JiAn-HuA HuANG

College of Science

National University of Defense Technology

Changsha, 410083, Hunan, P. R. China

E-mail address: jhhuang32@nudt.edu.cn 